\title{
Interferência da coloração de esmaltes de unha e do tempo na oximetria de pulso em voluntários sadios
}

\author{
Interference of nail polish colors and time \\ on pulse oximetry in healthy volunteers
}

Mara harumi MiYake ${ }^{1}$, Solange DicCinI², Ana Rita de Cássia BetTenCOURT ${ }^{2}$

\begin{abstract}
Introdução: A oximetria de pulso é um método não invasivo de mensuração da saturação periférica da oxiemoglobina $\left(\mathrm{SpO}_{2}\right)$. É freqüentemente utilizado em unidades de emergência, de terapia intensiva e em centro cirúrgico. A leitura da oximetria de pulso tem acurácia limitada na presença de metaemoglobina, carboxiemoglobina, anemia, vasoconstrição periférica, esmalte de unha, luz fluorescente e movimentação.
\end{abstract}

Objetivo: Avaliar a interferência da coloração de esmalte de unha e do tempo sobre a $\mathrm{SpO}_{2}$ em indivíduos sadios.

Método: Participaram do estudo 61 voluntárias sadias, com idades entre 18 e 32 anos. Foi avaliada $\mathrm{SpO}_{2}$ nas seguintes colorações de esmaltes: base (dedo mínimo), rosa claro (dedo anular), rosa claro com cintilante (dedo médio) e vermelha (polegar). 0 indicador não recebeu esmalte. Foi analisado o tempo para cada coloração de esmalte, a cada minuto até completar cinco minutos.

Resultados: Q uando comparadas as medidas da $\mathrm{SpO}_{2}$ com o controle, as colorações base $(p=0,56)$, rosa claro $(p=$ $0,56)$ e rosa claro com cintilante $(p=0,37)$ não apresentaram diferença estatisticamente significante. $\mathrm{A} \mathrm{SpO}_{2}$ apresentou variação significante na cor vermelha ( $p<0,001)$, porém manteve-se dentro dos parâmetros de normalidade. Não houve variação significativa da $\mathrm{SpO}_{2}$ ao longo do tempo.

Conclusão: A pesar da diferença encontrada com o uso de esmalte vermelho, todas as medidas da $\mathrm{SpO}_{2}$ obtidas encontraram-se dentro do intervalo de normalidade, para indivíduos normais. A análise de cada cor de esmalte de unha em relação ao tempo mostrou que não houve variação na leitura da $\mathrm{SpO}_{2}$. (J Pneumol 2003;29(6):386-90)

Descritores - Esmalte de unha. Saturação de oxigênio. Oximetria de pulso.
Background: Pulse oximetry is a noninvasive method to measure the saturation of peripheral oxyhaemoglobin $\left(\mathrm{SpO}_{2}\right)$. It's usually used in emergency, intensive care and operating room units. Pulse oximeter readings have limited accuracy in the presence of methemoglobin, carboxyhemoglobin, anaemia, peripheral vasoconstriction, nail polish, fluorescent light, and motion.

O bjectives: To evaluate the interferences of the color of nail polishes and time on $\mathrm{SpO}_{2}$ in healthy individuals.

Methods: Sixty-one healthy female volunteers, ages ranging from 18 to 32 years. The nail polish colors used to evaluate $\mathrm{SpO}_{2}$ were: base coat on the little finger, light pink on the ring finger, sparkling light pink on the medium finger and red on the thumb. The index finger was used as control and thus, did not receive nail polish. The time for each color was evaluated every minute until 5 minutes were completed.

Results: When the $\mathrm{SpO}_{2}$ measurement was compared with the control, the base coat $(p=0.56)$, light pink ( $p=$ $0.56)$ and sparkling light pink $(p=0.37)$ colors didn't present statistically significant differences. O nly the red presented a significant variation $(p<0,001)$, however it was within normal parameters. $\mathrm{SpO}_{2}$ didn't vary significantly with time.

Conclusion: Despite the difference found with the red color, all the $\mathrm{SpO}_{2}$ values achieved were inside the normal range for a healthy individual. Pulse oximeter readings are not significantly affected by the color of nail polish in relation to time.

Key words - Nail polish. Oxygen saturation. Pulse oximetry.
1. Enfermeira. Especialista em Terapia Intensiva e Enfermagem Cirúrgica. Bolsista da Disciplina Fundamentos de Enfermagem e Enfermagem Médico-Cirúrgica, Departamento de Enfermagem da Universidade Federal de São Paulo (U nifesp).

2. Enfermeira. Doutora em Ciências Pneumológicas. Professora Adjunta da Disciplina de Fundamentos de Enfermagem e Enfermagem
M édico-Cirúrgica do Departamento de Enfermagem da Universidade Federal de São Paulo (U nifesp).

Endereço para correspondência - Rua do Arraial, 80, apto. 147 04122-030 - São Paulo, SP. Tel.: (11) 5085-0277; e-mail: maraito@ terra.com.br

Recebido para publicação em 11/6/03. Aprovado, após revisão, em 15/10/03. 


\section{INTRODUÇÃO}

A oximetria de pulso é um método não invasivo de mensuração da saturação de oxigênio da hemoglobina arterial $\left(\mathrm{SpO}_{2}\right)$ e da pulsação cardíaca. ${ }^{(1,2)} \mathrm{O}$ desenvolvimento da oximetria de pulso tem sido baseado em mais de 100 anos de antecedentes tecnológicos e experimentais. ${ }^{(3)}$

A oximetria de pulso é muito utilizada para pacientes que necessitam de monitoramento contínuo da saturação de oxigênio. É amplamente utilizada em diversos locais, tais como: unidades de internação, ambulatório de teste da função pulmonar, pronto atendimento, terapia intensiva, centro cirúrgico e home care. É um método não invasivo, seguro, tem baixo custo, não necessita de pessoal especializado e apresenta resposta em curto período de tempo. ${ }^{(3)}$

Os princípios utilizados pelo método são a espectrofotometria, ou seja, oxiemoglobina e dioxiemoglobina diferem na absorção da luz vermelha e infravermelha; e a pletismografia, em que o volume de sangue arterial nos tecidos e a absorção de luz por esse sangue se alteram durante a pulsação. 0 oxímetro de pulso determina a saturação periférica de oxigênio pela hemoglobina $\left(\mathrm{SpO}_{2}\right)$ emitindo luz vermelha $(660 \mathrm{~nm})$ e infravermelha $(940 \mathrm{~nm})$ pelo leito arteriolar e medindo as mudanças na absorção de luz durante o ciclo pulsátil. U tiliza um sensor que possui, em um lado, um fotoemissor de luzes vermelha e infravermelha, denominado diodos emissores de luz (LED) e, no lado oposto, um fotorreceptor. ${ }^{(4)}$

A transmissão de luz através da pele, tecidos, veias e capilares é constante, e com a pulsação arterial o sangue oxigenado entra no tecido, alterando as suas características de reflexão e absorção de luz. A hemoglobina saturada por oxigênio absorve mais a luz infravermelha, enquanto a hemoglobina dessaturada absorve mais luz vermelha. A diferença entre os dois componentes de luz absorvida pulsátil (arterial) e não pulsátil (venosa) é continuamente analisada por um microprocessador, que calcula a saturação da hemoglobina do sangue arterial, eliminando, assim, os efeitos de absorventes não pulsáteis, como tecidos, ossos e sangue venoso. ${ }^{(1,3,4)}$

A pressão parcial de oxigênio no sangue arterial $\left(\mathrm{PaO}_{2}\right)$ tem decréscimo linear com a idade, devido ao aumento da diferença entre a perfusão e a ventilação. Os valores da $\mathrm{PaO}_{2}$ para indivíduos com 20 anos, 25 anos, 30 anos, 40 anos e com 50 anos está em torno de $100 \mathrm{mmHg}$, $98 \mathrm{mmHg}, 96 \mathrm{mmH} \mathrm{g}, 91 \mathrm{mmH} \mathrm{g}$ e de $87 \mathrm{mmHg} .{ }^{(5)}$

Os valores normais da $\mathrm{SpO}_{2}$ em ar ambiente, isto é, para uma fração inspirada de oxigênio $\left(\mathrm{FiO}_{2}\right)$ próxima a $21 \%$, estão entre $96 \%$ e $100 \%$, correspondendo a $\mathrm{PaO}_{2}$ em torno de 90 a $100 \mathrm{mmHg}$. A SpO 2 entre $91 \%$ e $92 \%$
Siglas e abreviaturas utilizadas neste trabalho

$\mathrm{COHb}$ - Carboxiemoglobina

$\mathrm{FiO}_{2}$ - Fração inspirada de oxigênio

$\mathrm{MetH} \mathrm{b}$ - Metaemoglobina

nm - Nanômetro

$\mathrm{PaO}_{2}$ - Pressão parcial de oxigênio

$\mathrm{SatO}_{2}$ - Saturação de oxigênio

T0 - Tempo zero

T1 - Tempo 1 (um minuto)

T2 - Tempo 2 (dois minutos)

T3 - Tempo 3 (três minutos)

T4 - Tempo quatro (quatro minutos)

T5 - Tempo cinco (cinco minutos)

é mantida quando a $\mathrm{PaO}_{2}$ está em torno de $60 \mathrm{mmHg}$, com desvio padrão de $\pm 3 \%$. . $^{(5)}$

Os fatores que interferem na oximetria de pulso são: falta de acurácia em situação em que a saturação de hemoglobina está abaixo de 70\%; carboxiemoglobina $(\mathrm{COHb})$, hemoglobina que tem maior afinidade ao monóxido de carbono, diminuindo a ligação ao oxigênio; metaemoglobina (MetH b), oxidação do íon ferroso à forma férrica impede que a hemoglobina se ligue ao oxigênio; corantes intravasculares (azul de metileno, indocianino verde, índigo vermelho); pigmentação da pele; esmaltes de unha; luz ambiente (fluorescentes, lâmpadas cirúrgicas, instrumentos fibroscópicos); movimentação do sensor; sensor não compatível com o aparelho; estados de choque em que há má perfusão tecidual; desvios da curva de saturação da hemoglobina (presença da pulsação venosa nas digitais); erros de leitura devidos a elevação dos níveis séricos de lipídios e bilirrubina alterando artificialmente os níveis de $\mathrm{COH}$ b e MetH b séricos e falta de calibração do aparelho. ${ }^{(2,3,5)}$

Segundo Wahr et al.,(5) os esmaltes de unhas de diversas marcas e cores podem alterar a saturação de oxigênio. A interferência na leitura da oximetria de pulso depende da absorção das luzes vermelha e infravermelha, resultando em decréscimo de $3 \%$ a $5 \%$ na $\mathrm{SpO}_{2}$.

Há poucas referências bibliográficas sobre a relação entre o esmalte de unha e a oximetria de pulso, sendo que a maioria é de revisões da literatura e poucos estudos são prospectivos clínicos. ${ }^{(6,7)}$

$\mathrm{Na}$ prática clínica, a retirada do esmalte de unha nem sempre é realizada devido a situações de emergência ou urgência do encaminhamento da paciente do pronto-socorro para o centro cirúrgico ou nas cirurgias ambulatoriais, em que a paciente muitas vezes é internada horas antes do procedimento cirúrgico. Existem poucos artigos, ${ }^{17-}$ 9) principalmente na literatura nacional, que pudessem, a partir de evidências científicas, comprovar ou não a interferência do esmalte de unha na leitura da oximetria de 
pulso. A partir desses questionamentos, foi realizado este estudo com o objetivo de avaliar a interferência da coloração de esmalte de unha e do tempo sobre a $\mathrm{SpO}_{2}$ em indivíduos sadios.

\section{Metodologia}

Foram estudadas 61 voluntárias sadias, com idade entre 18 e 35 anos, pertencentes ao grupo de alunas do curso de graduação em enfermagem da U niversidade Federal de São Paulo (U nifesp). Foram utilizados como critérios de exclusão: antecedentes patológicos cardíacos, pulmonares, hematológicos, distúrbios metabólicos como de lipídios e bilirrubina ${ }^{(3,5)}$ e a utilização prévia de esmalte de unha. Todas as voluntárias foram informadas sobre os objetivos do trabalho e após seu consentimento foi aplicado um instrumento de coleta de dados. Este trabalho foi autorizado pelo comitê de ética da instituição.

A coleta de dados foi realizada de novembro a dezembro de 2000, no laboratório do Departamento de Enfermagem da U nifesp. Foi utilizado um instrumento de coleta de dados, no qual foram anotados o número e as iniciais de cada voluntária, idade em anos e as medidas da $\mathrm{SpO}_{2}$ para cada coloração de esmalte utilizada neste estudo.

Antes do início da coleta de dados, foi realizado um pré-teste em 10 voluntárias sadias, em que foi verificada a $\mathrm{SpO}_{2}$ em todos os dedos da mão esquerda, sem esmalte. Observamos que não houve alteração da $\mathrm{SpO}_{2}$ entre os mesmos.

No laboratório de enfermagem, cada voluntária era acomodada em cadeira por 10 minutos, período este utilizado para a passagem de cada cor de esmalte e a secagem do mesmo. As colorações escolhidas correspondem às mais utilizadas em nosso meio. As colorações de esmalte utilizadas eram da marca Colorama ${ }^{\circledR}$ e foram aplicadas na mão esquerda obedecendo às seguintes seqüências: o dedo mínimo recebeu a cor base, o dedo anular a cor rosa claro, o dedo médio a cor rosa claro com cintilante, 0 indicador foi considerado o dedo controle, não sendo colocado nenhum esmalte de unha, e o polegar, a cor vermelha. A passagem de cada cor de esmalte de unha foi realizada da seguinte maneira: a primeira camada de esmalte de unha foi feita da esquerda (dedo mínimo) para a direita (dedo polegar) e, após o término, retornou-se ao dedo mínimo; iniciava-se a passagem da segunda camada do esmalte de unha com a coloração respectiva para cada dedo, no total de duas camadas de esmalte. 0 tempo total de secagem foi de sete minutos, utilizando secador de cabelo profissional, em potência máxima com ar frio, até a secagem completa do esmalte de unha. A distância utilizada entre o secador de cabelo e a mão foi de $10 \mathrm{~cm}$.

Para a mensuração da oximetria de pulso foi utilizado o aparelho portátil da marca Dixtal DX-2405 (Dixtal Biomédica Ind. Com. Ltda., São Paulo), com o sensor do tipo jacaré. Foram anotados os dados referentes a $\mathrm{SpO}_{2}$, correlacionando com as colorações de cada esmalte de unha. As anotações dos dados foram realizadas, respeitando-se a mesma seqüência da passagem dos esmaltes.

$\mathrm{Na}$ segunda parte da coleta de dados foi estudada a variável tempo para cada coloração do esmalte de unha. A primeira medida da $\mathrm{SpO}_{2}$ foi considerada como tempo zero (T 0$)$, tempo um (T 1), um minuto após o início da mensuração, e assim sucessivamente, até o tempo de cinco minutos (T5), num total de seis medidas. 0 tempo foi controlado por meio de um cronômetro digital Ari-timer Unicef 0601.

Para análise estatística da medida $\mathrm{SpO}_{2}$ para cada tipo de coloração de esmalte foi utilizada a prova de Wilcoxon e da $\mathrm{SpO}_{2}$ ao longo do tempo, a prova de Friedman.

\section{Resultados}

A média de idade das 61 voluntárias sadias foi de 22 anos (desvio padrão $=4$ anos), com idades variando entre 18 e 32 anos.

\section{Análise do efeito da presença do esmalte}

$\mathrm{Na}$ Tabela 1 são ap resentadas as análises comparativas da saturação de oxigênio $\left(\mathrm{SpO}_{2}\right)$ para cada esmalte em relação à condição controle.

TABELA 1

Medidas descritivas da saturação de oxigênio $\left(\mathrm{SpO}_{2}\right)$ para cada tipo de coloração de esmalte

\begin{tabular}{lcccccc}
\hline Coloração de esmalte & \multicolumn{4}{c}{ Saturação de oxigênio (\%) } & & \begin{tabular}{c} 
Prova de Wilcoxon \\
(comparação \\
\cline { 2 - 5 }
\end{tabular} \\
\cline { 2 - 5 } & Média & d.p. & Mediana & Mínimo & Máximo & \\
\hline Controle & 97,85 & 0,51 & 98 & 97 & 99 & \\
Base & 97,82 & 0,50 & 98 & 96 & 99 & $\mathrm{p}=0,564$ \\
Rosa claro & 97,89 & 0,49 & 98 & 97 & 99 & $\mathrm{p}=0,564$ \\
Rosa claro e cintilante & 97,80 & 0,51 & 98 & 97 & 99 & $\mathrm{p}=0,366$ \\
Vermelho & 97,26 & 0,68 & 97 & 96 & 99 & $\mathrm{p}<0,001^{*}$ \\
\hline
\end{tabular}


$\mathrm{Na}$ comparação entre as unhas com a cor base e a condição controle não foi encontrada diferença estatisticamente significante quanto à média de $\mathrm{SpO}_{2}(\mathrm{p}=0,56)$. H ouve concordância na medida de $\mathrm{SpO}_{2}$ em $49(80,3 \%)$ dos 61 casos, em cinco $(8,2 \%)$ casos a medida obtida no dedo com unha esmaltada de base foi maior em $1 \%$ e em sete $(11,5 \%)$ casos a medida obtida no dedo com unha esmaltada foi menor em $1 \%$.

$\mathrm{Na}$ comparação entre as unhas com esmalte rosa claro e a condição controle não foi encontrada diferença estatisticamente significante quanto à média de $\mathrm{SpO}_{2}(\mathrm{p}=$ $0,56)$. Houve concordância na medida de $\mathrm{SpO}_{2}$ em 49 $(80,3 \%)$ dos 61 casos, em sete $(11,5 \%)$ casos a medida obtida no dedo com unha esmaltada de rosa claro foi maior em $1 \%$ e em cinco $(8,2 \%)$ casos a medida obtida no dedo com unha esmaltada foi menor em $1 \%$.

$\mathrm{Na}$ comparação entre as unhas com esmalte rosa claro com cintilante e em condição controle não foi encontrada diferença estatisticamente significante quanto à média de $\mathrm{SpO}_{2}(\mathrm{p}=0,37)$. Houve concordância na medida de $\mathrm{SpO}_{2}$ em 50 (82\%) dos 61 casos, em quatro $(6,6 \%)$ casos a medida obtida no dedo com unha esmaltada de rosa claro com cintilante foi maior em $1 \%$ e em sete $(11,5 \%)$ casos a medida obtida no dedo com unha esmaltada foi menor em $1 \%$.

$\mathrm{Na}$ comparação entre as unhas com esmalte vermelho e em condição controle foi encontrada diferença estatisticamente significante quanto à média de $\mathrm{SpO}_{2}(\mathrm{p}<0,001)$, indicando que a média de $\mathrm{SpO}_{2}$ medida nas unhas esmaltadas de vermelho foi significantemente menor do que a medida das unhas de condição controle. Houve concordância na medida de $\mathrm{SpO}_{2}$ em $28(45,9 \%)$ dos 61 casos, em um $(1,6 \%)$ caso a medida obtida no dedo com unha esmaltada de vermelho foi maior em 1\%, em 28 (45,9\%) casos a medida obtida no dedo com unha esmaltada foi menor em $1 \%$, em três $(4,9 \%)$ casos a medida obtida no dedo com unha esmaltada foi menor em $2 \%$ e em um $(1,6 \%)$ caso a medida obtida no dedo com unha esmaltada foi menor em $3 \%$.

A pesar da diferença encontrada com o uso de esmalte vermelho, todas as medidas obtidas da $\mathrm{SpO}_{2}$ encontravamse dentro do intervalo de normalidade, de $96 \%$ a $99 \%$.

\section{Análise do efeito do tempo}

A análise referente ao tempo de exposição a cada condição de avaliação mostrou que não houve nenhum tipo de variação da $\mathrm{SpO}_{2}$ nas seis medidas tomadas durante os cinco minutos de seguimento $(\mathrm{T} 0, \mathrm{~T} 1, \mathrm{~T} 2, \mathrm{~T} 3, \mathrm{~T} 4 \mathrm{e}$ T5). As seis medidas foram idênticas para todas as 61 voluntárias em todas as condições de avaliação. As médias obtidas da $\mathrm{SpO}_{2}$ para controle, base, rosa claro e rosa claro com cintilante foram de $98 \%$ e para a cor vermelha, de $97 \%$ (Tabela 2).
TABELA 2

\begin{tabular}{|c|c|c|c|c|c|c|c|}
\hline oloração de esmalte & TO & T1 & T2 & T3 & T4 & T5 & $p^{\S}$ \\
\hline Controle & 98 & 98 & 98 & 98 & 98 & 98 & 1,000 \\
\hline 3ase & 98 & 98 & 98 & 98 & 98 & 98 & 1,000 \\
\hline Rosa claro & 98 & 98 & 98 & 98 & 98 & 98 & 1,000 \\
\hline Rosa claro e cintilante & 98 & 98 & 98 & 98 & 98 & 98 & 1,000 \\
\hline lermelho & 97 & 97 & 97 & 97 & 97 & 97 & 1,000 \\
\hline
\end{tabular}

s: Prova de Friedman

\section{DISCUSSÃO}

A oximetria de pulso é recomendada para qualquer paciente que tenha risco para hipoxemia, por ser um método que detecta precocemente a dessaturação, possui boa precisão e é de fácil utilização. ${ }^{(10)}$

Alterações nos valores da $\mathrm{SpO}_{2}$ devem ser cuidadosamente avaliadas. Vários fatores podem interferir na eficácia da leitura. Os principais são - limitações técnicas: luz do ambiente, ${ }^{(11)}$ posicionamento e localização do sensor (dedo da mão, do pé, lóbulo da orelha) $)^{(12)}$ e movimentação do paciente; ${ }^{(13,14)}$ limitações fisiológicas: pigmentação da pele, ${ }^{(15)}$ onicomicose, ${ }^{(16)}$ forma da curva de dissociação do oxigênio, ${ }^{(3,5)}$ presença de carboxiemoglobina ${ }^{(17)}$ e metaemoglobina, ${ }^{(18)}$ corantes como azul de metileno(19), baixa perfusão periférica(20) e esmalte de unha. ${ }^{(7)}$

Existem poucos estudos sobre os efeitos do uso de esmalte de unhas na mensuração da $\mathrm{SpO}_{2}$ através da oximetria de pulso. ${ }^{(7-9)}$. Kataria e Lampkins ${ }^{(8)}$ analisaram 15 voluntários e relataram que não houve interferência na presença de esmalte de unha; contudo, não especificaram as cores utilizadas.

No estudo realizado por Coté et al.,(7) 14 voluntários foram analisados em relação à interferência do esmalte na leitura da oximetria. Os esmaltes de unha de coloração preta, marrom escuro, azul e verde produziram significante queda na leitura da saturação de oxigênio e as colorações como vermelho e vinho não demonstraram alteração na leitura da oximetria de pulso. Nesse estudo, foram utilizados esmaltes de unha de marcas americanas, porém, não foi especificada a idade da população estudada, possíveis antecedentes patológicos, o tempo de estabilização para a realização da medida da saturação de oxigênio e situação de exercício ou repouso em que o indivíduo foi avaliado.

Contrariando os resultados do estudo anterior, Brand et al. ${ }^{(9)}$ analisaram 12 voluntários não fumantes e referiram que as cores azul, verde e verde limão não causaram diferença estatisticamente significante na oximetria de pulso.

Em nosso estudo, utilizamos as cores base, rosa claro, rosa claro com cintilante e vermelho e os resultados mos- 
traram que somente a cor vermelha apresentou interferência significativa na leitura da $\mathrm{SpO}_{2}$, porém, mantendo os valores dentro da normalidade, ou seja, $\mathrm{SpO}_{2}$ variando de $96 \%$ a $99 \%$.

A nalisando os estudos acima referidos, observamos que as cores de esmalte utilizadas, como preto, azul e verde, não correspondem às usadas no nosso meio, cuja tendência são as cores claras, nos tons rosa e bege ou vermelho e vinho. Uma das limitações do nosso estudo é que é impraticável testar todas as cores de esmaltes existentes no nosso meio.

O utra limitação a ser destacada é que seus resultados não podem ser extrapolados para indivíduos não sadios, como pacientes portadores de distúrbios cardiovasculares, hematológicos e pulmonares, entre outros.

\section{REFERÊNCIAS}

1. Knobel E. Condutas no paciente grave. $2^{a}$ ed. São Paulo: Editora A theneu, 1995;293-5.

2. Woodrow P. Pulse oximetry. Nursing Standard 1999;13:42-6.

3. Sinex JE. Pulse oximetry: principles and limitations. Am J Emerg Med 1999;17:59-66.

4. Carlson KA, Jahr J S. A historical overview and update on pulse oximetry. Anesthesiol Rev 1993;20:173-81.

5. Wahr J A, Tremper KK, Diab M. Pulse oximetry. Respir Care Clin N Am 1995; 1:77-105

6. Ralston AC, Webb RK, Runciman WB. Potential errors in pulse oximetry. III: Effects of interferences, dyes, dyshaemoglobins and other pigments. Anaesthesia 1991;46:291-5.

7. Coté CJ, Goldstein EA, Fuchsman WH, H oaglin DC. The effect of nail polish on pulse oximetry. Anesth Analg 1988;67:683-6.

8. Kataria BK, Lampkins R. Nail polish does not affect pulse oximeter saturation. A nesth A nalg 1986;65:824.

9. Brand TM, Brand ME, J ay GD. Enamel nail polish does not interfere with pulse oximetry among normoxic volunteers. J Clin Monit Comput 2002;17:93-6.

10. J ubran A. Pulse oximetry. Crit Care 1999;3:R11-R17.

11. Eisele J H, Downs D. A mbient light affects pulse oximetrers. A nesthesiology $1987 ; 67: 864-5$.
Interferência na mensuração da saturação de oximetria de pulso foi encontrada em alguns estudos, mas não em todos. Esse fato pode levar-nos a crer que a coloração da unha talvez não seja um bom preditor de interferência na oximetria e indica a necessidade de mais estudos sobre este assunto.

\section{CONCLUSÃO}

A pesar da diferença encontrada com o uso de esmalte vermelho em indivíduos normais, todas as medidas da $\mathrm{SpO}_{2}$ obtidas encontravam-se dentro do intervalo de normalidade.

A análise de cada cor de esmalte de unha em relação ao tempo mostrou que não houve variação na leitura da $\mathrm{SpO}_{2}$.

12. Grap MJ. Protocols for practice: applying research at the bedside. Critical Care Nurse 1998;18:94-9.

13. Poets $C F$, Stebbens VA. Detection of movement artifact in recorded pulse oximeter saturation. Eur J Pediatr 1997;156:808-11.

14. Plummer J L, Zakaria AZ, Ilsley AH, Fronsko RRL, O wen H. Evaluation of the influence of movement on saturation readings from pulse oximeters. Anaesthesia 1995;50:423-6.

15. Adler J N, Hughes LA, Vivilecchia R, Camargo CA J r. Effect of skin pigmentation on pulse oximetry accuracy in the emergency department. A cad Emerg Med 1998;5:965-70.

16. Ezri T, Szmuk P. Pulse oximeters and onychomycosis. A nesthesiology 1992;76:153-4.

17. Barker SJ , Tremper KK. The effect of carbon monoxide inhalation on pulse oximeter signal detection. A nesthesiology 1987;66:677-9.

18. Barker SJ , Tremper KK, H yatt J, Zaccari J . Effects of methemoglobinemia on pulse oximetry and mixed venous oximetry. A nesthesiology 1989;70:112-7.

19. Saito S, Fukura H, Shimada H, Fujita T. Prolonged interference of blue dye "patent blue" with pulse oximetry readings. Acta A naesthesiol Scand 1995;39:268-9.

20. Lindberg LG, Lennmarken C, Vegfors M. Pulse oximetry - clinical implications and recent technical developments. Acta Anaesthesiol Scand 1995;9:279-87. 